Review

\title{
Food Insecurity Is Associated with Cognitive Function: A Systematic Review of Findings across the Life Course
}

\author{
Michael F. Royer ${ }^{1}\left(\mathbb{D}\right.$, Nicolas Guerithault $^{1}$, B. Blair Braden ${ }^{1}$, Melissa N. Laska ${ }^{2}$ and Meg Bruening ${ }^{1, *} \mathbb{D}$ \\ 1 College of Health Solutions, Arizona State University, 550 N 3rd St, Phoenix, AZ 85004, USA; \\ mfroyer@asu.edu (M.F.R.); nguerith@asu.edu (N.G.); bbbraden@asu.edu (B.B.B.) \\ 2 Unit 300, Division of Epidemiology and Community Health, University of Minnesota, 1300 S 2nd St., \\ Minneapolis, MN 55454, USA; mnlaska@umn.edu \\ * Correspondence: meg.bruening@asu.edu
}

check for

updates

Citation: Royer, M.F.; Guerithault,

N.; Braden, B.B.; Laska, M.N.;

Bruening, M. Food Insecurity Is

Associated with Cognitive Function:

A Systematic Review of Findings

across the Life Course. Int. J. Transl.

Med. 2021, 1, 205-222.

https://doi.org/10.3390/

ijtm1030015

Academic Editor: Pier Paolo Claudio

Received: 13 August 2021

Accepted: 2 November 2021

Published: 9 November 2021

Publisher's Note: MDPI stays neutral with regard to jurisdictional claims in published maps and institutional affiliations.

Copyright: (c) 2021 by the authors. Licensee MDPI, Basel, Switzerland. This article is an open access article distributed under the terms and conditions of the Creative Commons Attribution (CC BY) license (https:/ / creativecommons.org/licenses/by/ $4.0 /)$.

\begin{abstract}
Food insecurity (FI) has negative implications across the life course that include poor health outcomes among both children and adults. However, the behavioral mechanisms by which FI impacts health behaviors are not clear. By understanding how FI is related to cognitive function/brain structure across the life course, we can design more targeted interventions. A systematic literature review was performed by conducting comprehensive database searches in Google Scholar and PubMed. Inclusion criteria required studies to include measures of FI and cognitive function/brain structure in humans. Study sample, design, outcomes, and biases were extracted. In total, 17 studies met the inclusion criteria. Cognitive domains included general cognition $(n=13)$, executive function $(n=10)$, visuospatial abilities $(n=4)$, and verbal memory $(n=8)$. No studies examined brain structure. Most studies ( $88 \%$ ) indicated significant inverse associations between FI and cognitive function across all stages of the life course, particularly for general cognition and executive function. Significant inverse associations were observed between FI and either general cognition or executive function among children $(n=3)$ and adults $(n=12)$. All studies considered confounding variables; however, given that all were observational, no causality can be inferred from the findings. These findings indicate that FI is related to lower cognitive function across the life course. Research should explore how changes in food security status impacts cognitive function and brain structure to develop optimal FI interventions and improve cognitive health.
\end{abstract}

Keywords: food insecurity; hunger; cognitive function; cognition; systematic review

\section{Introduction}

Food insecurity involves reduced quality, variety, or desirability of the diet, and can also include disrupted eating patterns with reduced food intake [1]. In 2019, 10.5\% of households (13.7 million) in the U.S. experienced food insecurity [2]. Certain groups are at higher risk of experiencing food insecurity, including households with children [3], unmarried people [4], and communities of color [5].

Food insecurity has negative implications across the life course that include negative outcomes among both children and adults [6]. Childhood food insecurity and hunger are linked to poor general health outcomes [7,8]. Food insecurity during childhood may include the onset of mental health struggles with anxiety [9], depression [10], and even suicide ideation [10]. Behavior problems [11] and physical aggression [9] are also related to childhood experiences with food insecurity. Numerous research studies have shown that schoolchildren experiencing household food insecurity have impaired levels of academic performance [12-14]. Adults experiencing food insecurity and hunger also tend to suffer poor health outcomes including heart disease [15], diabetes [16], obesity [15], hypertension [15,16], and sleep problems [17]. Among children and parents, there has been a demonstrated bi-directional relationship between food insecurity and depression [9]. 
Poor mental health is also associated with food insecurity among adults $[10,18]$ through pathways such as stress [18], anxiety [9], and depression [9].

Extensive research has yielded study findings that elucidate how poverty-related circumstances, such as food insecurity experiences resulting from being low income, incur negative health outcomes among vulnerable children [19] and adults [20]. Past studies have explored the relationship between varying degrees of undernutrition and numerous cognitive and behavioral outcomes. Results indicate inverse associations between these proxy factors and poor cognitive faculty functioning outcomes [21-28]. Researchers have also examined how malnutrition impacts the brain by using magnetic resonance imaging [29] and electroencephalography $[29,30]$. For example, A case-control study of post-mortem children studied the unique effects of severe undernutrition on neural development, and cases had significantly altered structural development of neural cells when compared with healthy controls [31].

Despite different etiologies, eating disorders (a psychological condition related to health) and food insecurity (a sociological condition related to health) share deficits in food consumption that may produce similar cognitive function outcomes. Past research has determined that anorexia can result in cognitive deficits related to reduced brain volumes [32]. Other studies on eating disorders and cognitive function have yielded contradicting results, as one study found no severe cognitive function impairment among anorexic adolescent females [33], while other research suggested that bulimic and anorexic women incur negative effects on cognitive function [34].

Research on voluntary caloric restriction (e.g., intermittent fasting) has provided additional insights on how food intake impacts cognitive function. Past research findings indicate that periodic caloric restriction can either maintain [35] or impair cognitive function [36]. Additionally, the results from studies examining caloric deprivation are similar to those evaluating caloric restriction, with cognitive function maintained $[37,38]$ or impaired [39]. Some studies have shown working memory improvements with caloric restriction [40], while a systematic review of voluntary experimental fasting showed either impaired or maintained cognitive function among participants [41].

It is possible that food-insecure populations have an overabundance of cheap, less healthful foods. Research has indicated that populations at risk for food insecurity have the double burden of an inadequate intake of key nutrients combined with the overconsumption of high-calorie, low-nutrient foods (sometimes referred to as overnutrition), [42,43] which is often linked with an increased prevalence of obesity and other chronic diseases [44-46]. There is an ongoing movement within the scientific community to examine food insecurity more holistically as nutrition [47], because the quality of food is as important as the quantity of food to promote health and prevent disease [48]. Dietary quality, overnutrition, and obesity have been linked to food insecurity [49-52] and cognitive function [53-56]. However, the literature linking overnutrition and obesity to food insecurity and cognitive health does not seem to be not as consistent or robust as the literature on eating disorders and caloric restriction [51,57,58].

With all the relevant extant scholarly literature considered, additional research that can improve the knowledge base regarding the link between food insecurity and cognitive function is essential. Therefore, we conducted a systematic review to more precisely understand this association by specifically focusing on the relationship between food insecurity and cognitive function. Given that cognitive function changes with age, a life course perspective is important for describing such patterns and transitions [59]. Another recent systematic review evaluated similar associations among middle-aged and older adults only and found (1) a positive association between early-life food insecurity and later-life cognitive function impairment, (2) a concurrent positive association between food insecurity and impaired cognitive function, and (3) a significantly faster decline in cognitive function among individuals experiencing the most severe form of food insecurity compared with their food-secure counterparts [60]. Our systematic review expands on $\mathrm{Na}$ et al.'s (2020) review by including studies with participants of all ages, while also 
examining the validity, bias, and statistical approaches used in each study [60]. We sought to broaden the understanding of how food insecurity and cognitive function are related by critically analyzing the nature of this relationship in study samples spanning the human life course. Understanding how food insecurity is related to cognitive function will help us to better understand the potential neurobiological associations of food insecurity across the life course.

\section{Materials and Methods}

We performed a systematic review guided by the Preferred Reporting Items for Systematic Reviews and Meta-Analyses (PRISMA) statement guidelines (Figure 1) to investigate the association between food insecurity and cognitive function. Specified inclusion criteria consisted of human studies involving subjects that were either currently or previously experiencing a condition related to food insecurity, which could also have consisted of food insufficiency, food shortage, and/or hunger. Included studies were required to have used a food insecurity measure and either a cognitive function or brain structure measure. Studies from any publication period were included. Exclusion criteria involved case studies; animal studies; and studies investigating malnutrition, eating disorders, or voluntary caloric restriction. We used these terms in the search to capture as many relevant studies as possible. However, while sharing similarities with food insecurity research, studies on malnutrition, eating disorders, and voluntary caloric restriction were excluded because a multitude of studies have already investigated the association between those variables and cognitive function [61-63].

Database searches were conducted in January 2021 using Google Scholar and PubMed to identify all relevant scholarly literature published in any year. The initial search terms for food insecurity (food insecurity, food insufficiency, food hardship, food shortage, hunger, malnourishment, malnutrition, undernutrition, food restriction, caloric restriction, food deprivation, caloric deprivation, eating disorders, and starvation) were paired with cognitive function terms (cognitive function, brain function, executive function, learning, decision making, memory, attention, mild cognitive impairment, psychological development, brain development, neurodevelopment, brain, neurology, neuroimaging, MRI, fMRI, EEG, and MEG). This process included 252 searches in both Google Scholar and PubMed, which totaled 504 searches (see Supplementary Materials Table S1). Two reviewers (MR, NG) screened the titles and abstracts of articles aligning with the selected key search terms, along with inclusion and exclusion criteria. Included studies were then thoroughly reviewed to confirm their inclusion.

A combination of two investigators reviewed each article (MB or MNL, paired with either MR or NG) to subsequently summarize and grade the literature. Key components of each study were summarized, which included the year the data were collected, country of study, dataset origin, sample size, study design, sex and age of study sample, food insecurity measure, cognitive function measure, brain measure, results summary, and confidence intervals. Confidence intervals are essential for validating the accuracy and precision of statistically significant findings [64]. As per the Preliminary Tool for Risk of Bias in Exposure Studies [65], Q-Coh [66], and other tools for grading the strength and quality of a body of evidence $[67,68]$, bias was assessed in each study by evaluating the following: the control and conditional groups were concurrently measured, similarity of groups at baseline, methods used to adjust or avoid selection bias, validity of measures, differential loss of subjects among groups, statistical power, recall bias, confidence interval statistics, missing information, discrepancies, and conflicts of interest. The PRISMA diagram (Figure 1) illustrates the research approach implemented for this systematic review. 


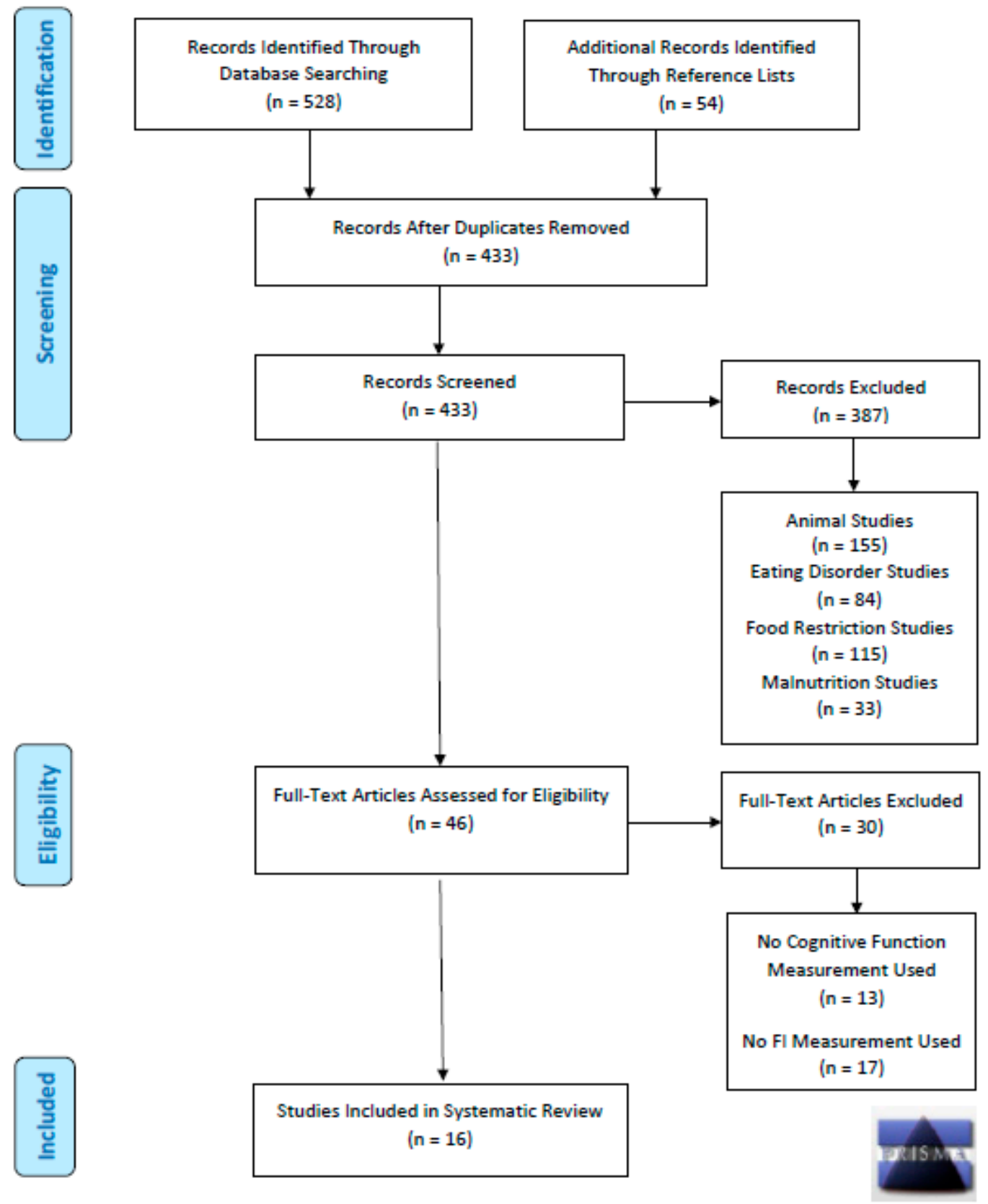

Figure 1. PRISMA Diagram- Search Strategy for Systematic Review on Food Insecurity and Cognitive Function.

\section{Results}

Participant age in the 17 included studies ranged from 9 months [69] to 85 years old [70], with sample sizes ranging from 97 [71] to 11,958 [72]. The 17 studies used a total of six food insecurity measures and 21 cognitive function measures. Observational study designs were used for all 17 studies (Table 1) [69-85]. A cross-sectional design was used in 14 studies [69-81,85], while all three of the longitudinal studies reported significant inverse associations between food insecurity and cognitive function over time [82-84]. 
Table 1. Characteristics, measures, and outcomes for studies examining food insecurity and cognitive function $(n=17)$.

\begin{tabular}{|c|c|c|c|c|c|c|c|c|}
\hline Authors & $\begin{array}{l}\text { Study } \\
\text { Design }\end{array}$ & $\begin{array}{l}\text { Year of } \\
\text { Data } \\
\text { Collection }\end{array}$ & Country & $\begin{array}{l}\text { Sample } \\
\text { Size }\end{array}$ & $\begin{array}{c}\text { Participant } \\
\text { Demographics }\end{array}$ & $\begin{array}{l}\text { Food Insecurity } \\
\text { Measures }\end{array}$ & $\begin{array}{l}\text { Cognitive } \\
\text { Function } \\
\text { Measures }{ }^{1}\end{array}$ & $\begin{array}{c}\text { Food Insecurity } \\
\text { and Cognitive } \\
\text { Function } \\
\text { Findings }^{2} \\
\end{array}$ \\
\hline $\begin{array}{l}\text { Alaimo } \\
\text { et al. } \\
(2001) \\
{[73]}\end{array}$ & $\begin{array}{l}\text { Observational, } \\
\text { cross- } \\
\text { sectional, } \\
\text { secondary } \\
\text { data }\end{array}$ & 1988-1994 & USA & 5349 & $\begin{array}{c}\text { Children; } \\
\text { aged 6-11 years } \\
\text { old } \\
\text { Adolescents; } \\
\text { aged 12-16 years } \\
\text { old }\end{array}$ & $\begin{array}{l}\text { NHANES III: } \\
\text { 'Sometimes or often } \\
\text { not enough food' }\end{array}$ & $\begin{array}{l}\text { WISC-R: Block } \\
\text { design and } \\
\text { digit span } \\
\text { tasks }\end{array}$ & $\begin{array}{l}\text { Food insecurity } \\
\text { and executive } \\
\text { func- } \\
\text { tion/visuospatial } \\
\text { abilities: (null) }\end{array}$ \\
\hline $\begin{array}{l}\text { Barnes } \\
\text { et al. } \\
(2012) \\
{[83]}\end{array}$ & $\begin{array}{l}\text { Observational, } \\
\text { longitudinal, } \\
\text { primary data }\end{array}$ & 1993-2009 & USA & 6105 & $\begin{array}{l}\text { Adults; mean } \\
74.9 \text { years old; } \\
61.8 \% \text { Black; } \\
60.7 \% \text { female }\end{array}$ & $\begin{array}{l}\text { One item asking } \\
\text { how often } \\
\text { participants went } \\
\text { without enough } \\
\text { food to eat during } \\
\text { childhood }\end{array}$ & MMSE & $\begin{array}{c}\text { Food insecurity } \\
\text { and general } \\
\text { cognition: } \\
\beta=-0.197 \\
p<0.001\end{array}$ \\
\hline $\begin{array}{l}\text { Cohn- } \\
\text { Schwartz } \\
\text { \& Wein- } \\
\text { stein } \\
(2020) \\
{[84]}\end{array}$ & $\begin{array}{l}\text { Observational, } \\
\text { longitudinal, } \\
\text { secondary } \\
\text { Data }\end{array}$ & 2009-2013 & $\begin{array}{l}\text { Europe } \\
\text { (Multi- } \\
\text { Country) }\end{array}$ & 2131 & $\begin{array}{l}\text { Adults; mean } \\
76.2 \text { years old; } \\
50 \% \text { female }\end{array}$ & $\begin{array}{l}\text { Two items: one } \\
\text { asking whether the } \\
\text { participant ever } \\
\text { experienced } \\
\text { hunger; one asking } \\
\text { when the hunger } \\
\text { period occurred }\end{array}$ & $\begin{array}{c}\text { RAVLT; } \\
\text { SHARE } \\
\text { immediate and } \\
\text { delayed word } \\
\text { recall; animal } \\
\text { naming task; } \\
\text { serial sevens } \\
\text { test }\end{array}$ & $\begin{array}{c}\text { Food insecurity } \\
\text { and impaired } \\
\text { general cognition } \\
\text { OR }=0.97 \\
95 \% \mathrm{CI}= \\
0.94-0.99 \text { and } \\
\text { verbal memory } \\
\beta=-1.23 \\
p=0.04\end{array}$ \\
\hline $\begin{array}{l}\text { Frith } \\
\text { and } \\
\text { Loprinzi } \\
(2018) \\
{[70]}\end{array}$ & $\begin{array}{l}\text { Observational, } \\
\text { cross- } \\
\text { sectional, } \\
\text { secondary } \\
\text { data }\end{array}$ & 1999-2002 & USA & 1851 & $\begin{array}{c}\text { Adults; } \\
\text { 60-85 years old }\end{array}$ & $\begin{array}{l}\text { USDA 18-item } \\
\text { Household Food } \\
\text { Security Survey } \\
\text { Module }\end{array}$ & DSST & $\begin{array}{c}\text { Food insecurity } \\
\text { and general } \\
\text { cognition: } \\
\beta=-14.4 ; \\
95 \% \text { CI }=-22.3 \\
-0.3\end{array}$ \\
\hline $\begin{array}{l}\text { Gao et al. } \\
\text { (2009) } \\
\text { [74] }\end{array}$ & $\begin{array}{l}\text { Observational, } \\
\text { cross- } \\
\text { sectional, } \\
\text { primary data }\end{array}$ & 2004-2009 & USA & 1358 & $\begin{array}{c}\text { Adults; } \\
45-75 \text { years old; } \\
70 \% \text { female }\end{array}$ & $\begin{array}{l}\text { USDA 10-item } \\
\text { Household Food } \\
\text { Security Scale }\end{array}$ & $\begin{array}{l}\text { MMSE; verbal } \\
\text { memory test; } \\
\text { digit span test }\end{array}$ & $\begin{array}{c}\text { Food insecurity } \\
\text { and executive } \\
\text { function } \\
\beta=-0.21 \\
p=0.003 \\
\text { and verbal } \\
\text { memory } \\
\beta=22.7 \\
p=0.04\end{array}$ \\
\hline $\begin{array}{l}\text { Grineski } \\
\text { et al. } \\
(2018) \\
{[72]}\end{array}$ & $\begin{array}{l}\text { Observational, } \\
\text { cross- } \\
\text { sectional, } \\
\text { secondary } \\
\text { data }\end{array}$ & 2010-2012 & USA & 11958 & $\begin{array}{c}\text { Children; } \\
\text { 5-9 years old; } \\
\text { 52\% White, 13\% } \\
\text { Black, 25\% } \\
\text { Hispanic, 4\% } \\
\text { Asian, } 6 \% \text { other }\end{array}$ & $\begin{array}{l}\text { USDA 18-item } \\
\text { Household Food } \\
\text { Security Survey } \\
\text { Module }\end{array}$ & $\begin{array}{l}\text { Numbers } \\
\text { reversed test; } \\
\text { 2-step } \\
\text { dimensional } \\
\text { change card } \\
\text { sort test }\end{array}$ & $\begin{array}{l}\text { Food insecurity } \\
\text { and executive } \\
\text { function: } \\
\beta=-5.20 \\
p \leq 0.05\end{array}$ \\
\hline $\begin{array}{l}\text { Hernandez } \\
\text { and Jac- } \\
\text { knowitz } \\
(2009) \\
\text { [69] }\end{array}$ & $\begin{array}{l}\text { Observational, } \\
\text { cross- } \\
\text { sectional, } \\
\text { secondary } \\
\text { data }\end{array}$ & 2001-2006 & USA & 7900 & $\begin{array}{c}\text { Children; } \\
\text { 9-24 months old }\end{array}$ & $\begin{array}{l}\text { USDA 18-item } \\
\text { Household Food } \\
\text { Security Survey } \\
\text { Module }\end{array}$ & $\begin{array}{l}\text { BSF-R mental } \\
\text { scale }\end{array}$ & $\begin{array}{c}\text { Food insecurity } \\
\text { and general } \\
\text { cognition: } \\
\beta=-1.62 \\
p<0.05\end{array}$ \\
\hline $\begin{array}{l}\text { Hobbs } \\
\text { and } \\
\text { King } \\
(2018) \\
{[75]}\end{array}$ & $\begin{array}{l}\text { Observational, } \\
\text { cross- } \\
\text { sectional, } \\
\text { secondary } \\
\text { data }\end{array}$ & 1998-2000 & USA & 2046 & $\begin{array}{c}\text { Children; } 5 \text { years } \\
\text { old; } 22.5 \% \text { White; } \\
52.6 \% \text { Black; } \\
21.6 \% \text { Hispanic; } \\
\text { 3.3\% other }\end{array}$ & $\begin{array}{l}\text { USDA 18-item } \\
\text { Household Food } \\
\text { Security Survey } \\
\text { Module }\end{array}$ & $\begin{array}{l}\text { Peabody: } \\
\text { PVT-R; } \\
\text { W-J } \\
\text { letter-word }\end{array}$ & $\begin{array}{c}\text { Food insecurity } \\
\text { and general } \\
\text { cognition: } \\
\beta=-0.19 \\
95 \% \text { CI }=-0.33 \\
-0.04\end{array}$ \\
\hline $\begin{array}{c}\text { Hobkirk } \\
\text { et al. } \\
(2017) \\
{[71]}\end{array}$ & $\begin{array}{l}\text { Observational, } \\
\text { cross- } \\
\text { sectional, } \\
\text { primary data }\end{array}$ & 2010-2014 & USA & 97 & $\begin{array}{c}\text { Adults; mean } \\
45 \text { years old; } 85 \% \\
\text { Black; } 35 \% \\
\text { female }\end{array}$ & $\begin{array}{l}\text { HFIAS for } \\
\text { Measurement of } \\
\text { Food Access: } \\
\text { Indicator Guide } \\
\text { Version } 3\end{array}$ & $\begin{array}{l}\text { WAIS-III; } \\
\text { HVLT; } \\
\text { BVMT-R; } \\
\text { Stroop test; } \\
\text { trail-making } \\
\text { test; } \\
\text { PASAT-100; } \\
\text { NAB digits } \\
\text { test; FAS; } \\
\text { grooved } \\
\text { pegboard test }\end{array}$ & $\begin{array}{l}\text { Food insecurity } \\
\text { and general cog- } \\
\text { nition/executive } \\
\text { function: } \\
\text { (null) }\end{array}$ \\
\hline
\end{tabular}


Table 1. Cont.

\begin{tabular}{|c|c|c|c|c|c|c|c|c|}
\hline Authors & $\begin{array}{l}\text { Study } \\
\text { Design }\end{array}$ & $\begin{array}{l}\text { Year of } \\
\text { Data } \\
\text { Collection }\end{array}$ & Country & $\begin{array}{l}\text { Sample } \\
\text { Size }\end{array}$ & $\begin{array}{c}\text { Participant } \\
\text { Demographics }\end{array}$ & $\begin{array}{l}\text { Food Insecurity } \\
\text { Measures }\end{array}$ & $\begin{array}{l}\text { Cognitive } \\
\text { Function } \\
\text { Measures }{ }^{1}\end{array}$ & $\begin{array}{c}\text { Food Insecurity } \\
\text { and Cognitive } \\
\text { Function } \\
\text { Findings }^{2}\end{array}$ \\
\hline $\begin{array}{l}\text { Koyanagi } \\
\text { et al. } \\
(2019) \\
\text { [76] }\end{array}$ & $\begin{array}{l}\text { Observational, } \\
\text { cross- } \\
\text { sectional, } \\
\text { secondary } \\
\text { data }\end{array}$ & 2007-2008 & $\begin{array}{l}\text { South } \\
\text { Africa }\end{array}$ & 3672 & $\begin{array}{l}\text { Adults; mean } \\
61.4 \text { years old; } \\
74.2 \% \text { Black, } 8.2 \% \\
\text { White, } 16.5 \% \\
\text { other; } 56 \% \\
\text { female }\end{array}$ & $\begin{array}{l}\text { Two items adapted } \\
\text { from NHANES }\end{array}$ & $\begin{array}{c}\text { CERAD; } \\
\text { WAIS-III; } \\
\text { Animal } \\
\text { Naming Task }\end{array}$ & $\begin{array}{c}\text { Food insecurity } \\
\text { and impaired } \\
\text { general } \\
\text { cognition: } \\
\text { OR }=2.41 \\
95 \% C I=1.63 \\
3.87\end{array}$ \\
\hline $\begin{array}{l}\text { Mayston } \\
\text { et al. } \\
(2015) \\
{[77]}\end{array}$ & $\begin{array}{l}\text { Observational, } \\
\text { cross- } \\
\text { sectional, } \\
\text { primary data }\end{array}$ & 2008-2010 & India & 1934 & $\begin{array}{c}\text { Adults; mean } \\
35 \text { years old; } 53 \% \\
\text { female }\end{array}$ & $\begin{array}{l}\text { One item asking if } \\
\text { participants had } \\
\text { 'ever experienced } \\
\text { hunger due to a } \\
\text { lack of money' }\end{array}$ & $\begin{array}{c}\text { Word-list } \\
\text { learning } \\
\text { memory task; } \\
\text { animal naming } \\
\text { verbal fluency } \\
\text { task }\end{array}$ & $\begin{array}{c}\text { Food insecurity } \\
\text { and impaired } \\
\text { verbal memory: } \\
O R=1.41 \\
95 \% C I=1.05 \\
1.88\end{array}$ \\
\hline $\begin{array}{c}\text { Momtaz } \\
\text { et al. } \\
(2015) \\
{[78]}\end{array}$ & $\begin{array}{l}\text { Observational, } \\
\text { cross- } \\
\text { sectional, } \\
\text { secondary } \\
\text { data }\end{array}$ & 2003-2005 & Malaysia & 2745 & $\begin{array}{l}\text { Adults; } 60+\text { years } \\
\text { old }\end{array}$ & $\begin{array}{l}\text { One item asking if } \\
\text { participants had } \\
\text { 'enough food to eat' }\end{array}$ & $\begin{array}{l}\text { GMS- } \\
\text { AGECAT: } \\
\text { Malaysian- } \\
\text { adapted }\end{array}$ & $\begin{array}{c}\text { Food insecurity } \\
\text { \& impaired } \\
\text { general } \\
\text { cognition: } \\
\text { OR }=1.81 \\
95 \% \text { CI }=1.13 \\
2.92\end{array}$ \\
\hline $\begin{array}{c}\text { Onadja } \\
\text { et al. } \\
(2013) \\
{[79]}\end{array}$ & $\begin{array}{l}\text { Observational, } \\
\text { cross- } \\
\text { sectional, } \\
\text { secondary } \\
\text { data }\end{array}$ & 2010 & $\begin{array}{l}\text { Burkina } \\
\text { Faso }\end{array}$ & 981 & $\begin{array}{l}\text { Adults; } 50+\text { years } \\
\text { old; } 52.6 \% \text { female }\end{array}$ & $\begin{array}{c}\text { Measure } \\
\text { examining 'food } \\
\text { availability } \\
\text { uncertainty,' 'food } \\
\text { intake reduction,' } \\
\text { and 'totally lacking } \\
\text { food' }\end{array}$ & LCT & $\begin{array}{c}\text { Food insecurity } \\
\text { and impaired } \\
\text { general } \\
\text { cognition: } \\
\text { OR }=1.80 \\
95 \% C I=1.06, \\
3.06\end{array}$ \\
\hline $\begin{array}{l}\text { Portela- } \\
\text { Parra } \\
\text { and } \\
\text { Leung } \\
(2019) \\
{[80]}\end{array}$ & $\begin{array}{l}\text { Observational, } \\
\text { cross- } \\
\text { sectional, } \\
\text { secondary } \\
\text { data }\end{array}$ & 2011-2014 & USA & 1823 & $\begin{array}{c}\text { Adults; } 60+\text { years } \\
\text { old; } 54 \% \text { female; } \\
42.7 \% \\
\text { Non-Hispanic } \\
\text { White, } 22.9 \% \\
\text { Non-Hispanic } \\
\text { Black; } 25.8 \% \\
\text { Hispanic, } 8.6 \% \\
\text { Non-Hispanic } \\
\text { other }\end{array}$ & $\begin{array}{l}\text { USDA 10-item } \\
\text { Adult Food } \\
\text { Security Survey } \\
\text { Module }\end{array}$ & $\begin{array}{l}\text { CERAD: word } \\
\text { learning } \\
\text { subtest, } \\
\text { delayed word } \\
\text { cecall; AFT, } \\
\text { DSST }\end{array}$ & $\begin{array}{c}\text { Food insecurity } \\
\text { and general } \\
\text { cognition } \\
\beta=-0.24 \\
95 \% C I=-0.33, \\
-0.15, \\
\text { executive } \\
\text { function } \\
\beta=-0.13 \\
95 \% C I=-0.25, \\
-0.002, \\
\text { and verbal } \\
\text { memory: } \\
\beta=-0.14 \\
95 \% C I=-0.26, \\
-0.01\end{array}$ \\
\hline $\begin{array}{l}\text { Tan et al. } \\
(2020) \\
\text { [85] }\end{array}$ & $\begin{array}{l}\text { Observational, } \\
\text { cross- } \\
\text { sectional, } \\
\text { secondary } \\
\text { data }\end{array}$ & 2013-2015 & USA & 1346 & $\begin{array}{c}\text { Adults; median } \\
\text { 49.6 years old; } \\
\text { 68.1\% Black, } \\
\text { 10.9\% White, } \\
\text { 16.4\% Hispanic; } \\
\text { 4.6\% other }\end{array}$ & $\begin{array}{l}\text { USDA 18-item } \\
\text { Household Food } \\
\text { Security Survey } \\
\text { Module }\end{array}$ & $\begin{array}{l}\text { Letter-number } \\
\text { span task, } \\
\text { Stroop test, } \\
\text { symbol digit } \\
\text { modalities test, } \\
\text { trail-making } \\
\text { test Part B, } \\
\text { HVLT, COWAT }\end{array}$ & $\begin{array}{l}\text { Food insecurity } \\
\text { \& executive } \\
\text { bunction: } \\
\beta=-1.45 \\
p \leq 0.01\end{array}$ \\
\hline $\begin{array}{l}\text { Tong } \\
\text { et al. } \\
(2018) \\
{[81]}\end{array}$ & $\begin{array}{l}\text { Observational, } \\
\text { cross- } \\
\text { sectional, } \\
\text { secondary } \\
\text { data }\end{array}$ & 2013-2014 & USA & 250 & $\begin{array}{c}\text { Adults; mean } \\
58 \text { years old; } \\
79.7 \% \text { Black, } \\
\text { 10.9\% White, } \\
4.6 \% \\
\text { Hispanic/Latino; } \\
22.9 \% \text { female }\end{array}$ & $\begin{array}{l}\text { USDA Household } \\
\text { Food Security } \\
\text { Survey Module } \\
\text { 6-item Short Form }\end{array}$ & MMSE & $\begin{array}{c}\text { Food insecurity } \\
\text { and impaired } \\
\text { general } \\
\text { cognition: } \\
\text { OR }=2.21 \\
95 \% C I=1.12 \\
4.35\end{array}$ \\
\hline
\end{tabular}


Table 1. Cont.

\begin{tabular}{|c|c|c|c|c|c|c|c|c|}
\hline Authors & $\begin{array}{l}\text { Study } \\
\text { Design }\end{array}$ & $\begin{array}{l}\text { Year of } \\
\text { Data } \\
\text { Collection }\end{array}$ & Country & $\begin{array}{l}\text { Sample } \\
\text { Size }\end{array}$ & $\begin{array}{c}\text { Participant } \\
\text { Demographics }\end{array}$ & $\begin{array}{l}\text { Food Insecurity } \\
\text { Measures }\end{array}$ & $\begin{array}{l}\text { Cognitive } \\
\text { Function } \\
\text { Measures }{ }^{1}\end{array}$ & $\begin{array}{l}\text { Food Insecurity } \\
\text { and Cognitive } \\
\text { Function } \\
\text { Findings }{ }^{2}\end{array}$ \\
\hline $\begin{array}{l}\text { Wong } \\
\text { et al. } \\
(2016) \\
{[82]}\end{array}$ & $\begin{array}{l}\text { Observational, } \\
\text { longitudinal, } \\
\text { primary data }\end{array}$ & 2004-2009 & USA & 597 & $\begin{array}{c}\text { Adults; } \\
45-75 \text { years old; } \\
68.3 \% \text { female }\end{array}$ & $\begin{array}{l}\text { USDA 10-item } \\
\text { Household Food } \\
\text { Security Survey } \\
\text { Module }\end{array}$ & $\begin{array}{l}\text { MMSE, } \\
\text { 16-word } \\
\text { learning list, } \\
\text { digit span test } \\
\text { (forward and } \\
\text { backward), } \\
\text { Stroop test, } \\
\text { verbal fluency } \\
\text { test, clock } \\
\text { drawing test, } \\
\text { figure copying }\end{array}$ & $\begin{array}{c}\text { Food insecurity } \\
\text { and general } \\
\text { cognition } \\
\beta=-1.20 \\
95 \% C I=-2.19, \\
-0.20, \\
\text { executive } \\
\text { function } \\
\beta=-4.67 \\
95 \% C I=-8.52, \\
-0.85, \text { and } \\
\text { visuospatial } \\
\text { abilities: } \\
\beta=-6.18 \\
95 \% C I=-8.92, \\
-3.43\end{array}$ \\
\hline
\end{tabular}

${ }^{1}$ FT: Animal Fluency Test; BSF-R: Bayley Short Form—Research; AUDIT: Alcohol Abuse Disorders Inventory Test; BVMT-R: Brief Visuospatial Memory Test-Revised; CERAD: Consortium to Establish a Registry for Alzheimer's Disease; COWAT: Controlled Oral Word Association Test; DSST: Digit Symbol Substitution Test; GMS-AGECAT: Geriatric Mental State-Automated Geriatric Examination for Computer-Assisted Taxonomy; HFIAS: Household FI Access Scale; HVLT: Hopkins Verbal Learning Test-Revised; LCT: Leganés Cognitive Test; MCI: Mild Cognitive Impairment; MMSE: Mini Mental State Examination; NAB: Neuropsychological Assessment Battery; NHANES: National Health and Nutrition Examination Survey; PASAT-100: Paced Auditory Serial Addition Task-100; Peabody PVT-R: Peabody Picture Vocabulary Test-Revised; RAVLT: Rey's Auditory Verbal Learning Test; SHARE: Survey of Health, Ageing, and Retirement in Europe; WAIS-III: Weschler Adult Intelligence Scale; WISC-R: Weschler Intelligence Scale for Children-Revised; W-J Letter-Word: Woodcock-Johnson Test of Achievement Letter-Word Identification Subtest ${ }^{2} \beta=$ standardized beta coefficient; $b=$ unstandardized beta $^{2}$ coefficient; $\mathrm{OR}=$ odds ratio; $\mathrm{CI}=$ confidence interval.

The findings from 15 of the 17 studies suggest a significant inverse association between food insecurity and cognitive function [69,70,72,74-85]. The significant inverse relationship between food insecurity and cognitive function was reported in three of the four studies among children [69,72,75] and 12 of the 13 studies among adults [70,74,76-85]. The odds ratios reported in five studies indicated that middle-aged and older adults experiencing food insecurity have significantly greater odds of demonstrating impaired general cognition compared with their food-secure counterparts [76,78,79,81,84].

Four studies assessed food insecurity and cognitive function in children [69,72,73,75]; ranging from toddlers (9-24 months) [69] to teens (13-16 years) [73]. Among children, food insecurity was reported to be inversely associated with general cognition $[69,75]$ and executive function [72]. Hernandez and Jacknowitz (2009) found that maternal food insecurity was negatively associated with children's general cognition $(p<0.05)$ at 2 years of age [69], while Hobbs and King (2018) reported similar findings among children who were 5 years old $(95 \%$ CI $-0.33,-0.44)$ [75]. Grineski et al. (2018) reported that food insecurity was negatively correlated with executive function $(p<0.05)$ among children aged 5-9 years old [72]. Contrarily, Alaimo et al. (2001) did not find significant impairment in executive functioning or visuospatial abilities in food-insecure children or teens [73].

A total of 13 studies recruited adult participant samples [70,71,74,76-85]. Among adults, food insecurity has been found to be inversely associated with general cognition $[70,76,78-84]$, executive function $[80,82,85]$, verbal memory $[77,80,84]$, and visuospatial abilities [82]. In some studies, this association was influenced by participant characteristics such as age [74,76], race [83], dementia status [78], or HIV status [71,85]. The study findings from research performed among Puerto Rican adults in the U.S. indicated a significant inverse trend between food security status and general cognitive function $(p=0.04)$, executive functioning $(p=0.006)$, and verbal memory $(p=0.01)$ [74]. The results from a multi-country study among older adults in Europe that measured reports of childhood hunger and cognitive function later in life reported significantly worse verbal memory $(p<0.04)$ outcomes among participants who experienced hunger between ages 0 and 
4 when compared with individuals with no past hunger experiences [84]. Additionally, a study of South African adults found that moderate and severe food insecurity were associated with 2.82 (95\% CI: 1.65-4.84) and 2.51 (95\% CI: 1.63-3.87) times greater odds of impaired general cognition [76].

Primary data analyses were performed in five reviewed studies $[71,74,77,82,83]$, while secondary data analyses were conducted in 12 studies $[69,70,72,73,75,76,78-81,84,85]$. A relatively diverse demographic has been studied in research investigating the link between food insecurity and cognition, as study samples have included younger to middle-aged Indian adults [77], middle-aged to older Puerto Rican adults [74,82], older Malaysian adults [78], a middle-aged sample of South African adults [76], and middle-aged Burkinabé adults [79]. As detailed in Table 2, the most frequently used measurement items for food security were derived from the United States Department of Agriculture's Household Food Security Scale [86], while cognitive function was most often examined with the Wechsler Adult Intelligence Scale-Third Edition [87]. Questions from the Household Food Security Scale were used in nine studies $[69,70,72,74,75,80-82,85]$, and items from the Wechsler Adult Intelligence Scale were used in three studies [71,76,80].

A thorough evaluation of the study biases and methodological strengths was conducted (Table 3). To adjust for bias, study researchers used a variety of approaches, including multistage probability design [70], stratified multistage cluster sampling design [76], oversampling of subgroups [69,73], sampling of matched controls [85], and sampling weights [72,73]. Recall bias was present in all studies, given that each was observational by design and included self-reported survey assessments of either food insecurity [69-85] and/or cognitive function [76]. Six studies adjusted for selection bias by obtaining a participant sample that was representative of the greater target population $[69,70,72,73,76,81]$. Strong measurement validity was determined for nine studies in their evaluations of food insecurity and cognitive function [69-72,74,75,79,80,82,85]. Seven studies had mixed measurement validity due to unreliable or limited food insecurity measures $[73,76-78,81,83,84]$. Studies were deemed to have mixed validity due to various factors including: severe recall bias derived from self-report measures of early-life childhood food insecurity among older adults who were assumed to have dementia [78], the use of a household food security scale with a homeless study sample [81], and food insecurity measures limited in scope using only one item $[73,77,83]$ or two items $[76,84]$. Moreover, significant $95 \%$ confidence intervals were reported for cognitive function outcomes in 11 studies [70,74-82,84], and all but two studies reported precise statistics $[70,82]$.

Table 2. Measures used across the life course by cognitive domain in studies exploring the mechanisms of food insecurity ${ }^{1,2}$.

\begin{tabular}{|c|c|c|c|c|}
\hline Citation. & General Cognition: & $\begin{array}{c}\text { Executive Functioning: } \\
\text { (Working Memory, Cognitive } \\
\text { Flexibility, Inhibition, } \\
\text { Planning, Reasoning) }\end{array}$ & $\begin{array}{l}\text { Visuospatial Abilities: } \\
\text { (Perception, } \\
\text { Construction) }\end{array}$ & $\begin{array}{c}\text { Verbal Memory: } \\
\text { (Short-Term, Long-Term) }\end{array}$ \\
\hline Alaimo et al. (2001) [73] & & WISC-R: digit span & WISC-R: block design & \\
\hline Barnes et al. (2012) [83] & MMSE ** & & & \\
\hline $\begin{array}{c}\text { Cohn-Schwartz \& } \\
\text { Weinstein (2020) [84] }\end{array}$ & Serial sevens test* & Animal naming task & & $\begin{array}{l}\text { RAVLT immediate recall } \\
\text { RAVLT delayed recall ** }\end{array}$ \\
\hline $\begin{array}{l}\text { Frith and Loprinzi (2018) } \\
\text { [70] }\end{array}$ & DSST *CI & & & \\
\hline Gao et al. (2009) [74] & MMSE * & $\begin{array}{c}\text { Digit span backward ** } \\
\text { Stroop test } \\
\text { Letter fluency * }\end{array}$ & $\begin{array}{l}\text { Figure copying test } \\
\text { Clock drawing test }\end{array}$ & $\begin{array}{l}\text { Digit span forward } \\
\text { Word list learning * } \\
\text { Word recognition } \\
\text { Word percentage retention * }\end{array}$ \\
\hline Grineski et al. (2018) [72] & & $\begin{array}{l}\text { Numbers reversed test } \\
\text { 2-step dimensional change } \\
\text { card sort test }\end{array}$ & & \\
\hline $\begin{array}{c}\text { Hernandez and } \\
\text { Jacknowitz (2009) [69] }\end{array}$ & BSF-R: cognitive scale & & & \\
\hline $\begin{array}{l}\text { Hobbs and King (2018) } \\
\text { [75] }\end{array}$ & $\begin{array}{l}\text { Peabody: PVT-R ** } \\
\text { W-J: letter-word * }\end{array}$ & & & \\
\hline
\end{tabular}


Table 2. Cont.

\begin{tabular}{|c|c|c|c|c|}
\hline Citation. & General Cognition: & $\begin{array}{c}\text { Executive Functioning: } \\
\text { (Working Memory, Cognitive } \\
\text { Flexibility, Inhibition, } \\
\text { Planning, Reasoning) }\end{array}$ & $\begin{array}{c}\text { Visuospatial Abilities: } \\
\text { (Perception, } \\
\text { Construction) }\end{array}$ & $\begin{array}{c}\text { Verbal Memory: } \\
\text { (Short-Term, Long-Term) }\end{array}$ \\
\hline Hobkirk et al. (2019) [71] & $\begin{array}{l}\text { WAIS-III: digit symbol } \\
\text { PASAT-100 } \\
\text { NAB digits } \\
\text { forward/digits } \\
\text { backward test }\end{array}$ & $\begin{array}{l}\text { Stroop test } \\
\text { Trail-making test Part B } \\
\text { FAS: letter fluency } \\
\text { FAS: category fluency }\end{array}$ & BVMT-R & HVLT \\
\hline Koyanagi et al. (2019) [76] & $\mathrm{MCI} * * *$ & $\begin{array}{l}\text { WAIS-III: digit span forward } \\
\text { and backward }{ }^{\mathrm{a}} \\
\text { Animal naming task }\end{array}$ & & $\begin{array}{l}\text { CERAD: word } \\
\text { learning a } \\
\text { CERAD: delayed } \\
\text { recall }^{\mathrm{a}}\end{array}$ \\
\hline Mayston et al. (2015) [77] & & Animal naming task: memory & & $\begin{array}{l}\text { Word list learning: delayed } \\
\text { recall * }\end{array}$ \\
\hline $\begin{array}{c}\text { Momtaz et al. (2015) [78] } \\
\text { Onadja et al. (2013) [79] }\end{array}$ & $\begin{array}{c}\text { GMS-AGECAT: } \\
\text { Malaysian-adapted ** } \\
\text { LCT *CI }\end{array}$ & & & \\
\hline $\begin{array}{l}\text { Portela-Parra and Leung } \\
\text { (2019) [80] }\end{array}$ & $\mathrm{DSST}^{*}$ & $\mathrm{AFT}^{*}$ & & $\begin{array}{c}\text { CERAD: word } \\
\text { learning * } \\
\text { CERAD: delayed recall }\end{array}$ \\
\hline Tan et al. (2020) [85] & & $\begin{array}{l}\text { Letter-number span task } \\
\text { Stroop test } \\
\text { a } \\
\text { Symbol digit modalities test } \\
\text { Trail-mMaking test Part } \mathrm{B}^{\mathrm{a}}\end{array}$ & & $\begin{array}{l}\text { HVLT }^{a} \\
\text { COWAT }^{a}\end{array}$ \\
\hline $\begin{array}{l}\text { Tong et al. (2018) [81] } \\
\text { Wong et al. (2016) [82] }\end{array}$ & $\begin{array}{l}\mathrm{MMSE} * \mathrm{CI} \\
\mathrm{MMSE} * \mathrm{CI}\end{array}$ & $\begin{array}{c}\text { Digit span backward } \\
\text { Stroop test } \\
\text { Letter fluency * }\end{array}$ & $\begin{array}{l}\text { Figure copying test } \\
\text { Clock drawing test }\end{array}$ & $\begin{array}{l}\text { Digit span forward } \\
\text { Word list learning } \\
\text { Word recognition }\end{array}$ \\
\hline
\end{tabular}

\footnotetext{
${ }^{1}$ AFT: Animal Fluency Test; BSF-R: Bayley Short Form—Research Edition; AUDIT: Alcohol Abuse Disorders Inventory Test; BVMT-R: Brief Visuospatial Memory Test-Revised; CERAD: Consortium to Establish a Registry for Alzheimer's Disease; COWAT: Controlled Oral Word Association Test; DSST: Digit Symbol Substitution Test; GMS-AGECAT: Geriatric Mental State-Automated Geriatric Examination for Computer-Assisted Taxonomy; HVLT-R: Hopkins Verbal Learning Test; LCT: Leganés Cognitive Test; MCI: Mild Cognitive Impairment; MMSE: Mini Mental State Examination; NAB: Neuropsychological Assessment Battery; NHANES: National Health and Nutrition Examination Survey; PASAT-100: Paced Auditory Serial Addition Task-100; Peabody PVT-R: Peabody Picture Vocabulary Test-Revised; RAVLT: Rey's Auditory Verbal Learning Test; SHARE: Survey of Health, Ageing, and Retirement in Europe; WAIS-III: Weschler Adult Intelligence Scale; WISC-R: Weschler Intelligence Scale for Children-Revised; W-J Letter-Word: Woodcock-Johnson Test of Achievement Letter-Word Identification Subtest ${ }^{2 *}=p \leq 0.05 ;{ }^{* *}=p \leq 0.01 ;{ }^{* * *}=p \leq 0.001 ;{ }^{* C I}=$ significant confidence interval; ${ }^{\text {a }}$ Statistical results not reported for specific test.
}

Table 3. Research methodology, study design, biases, measurement validity, and analytical approaches.

\begin{tabular}{|c|c|c|c|c|c|c|}
\hline Citation & $\begin{array}{l}\text { Study } \\
\text { Design }\end{array}$ & $\begin{array}{l}\text { Methods to Adjust } \\
\text { for Selection Bias }\end{array}$ & $\begin{array}{c}\text { Validity of } \\
\text { Measures } \\
{[+/-]}\end{array}$ & $\begin{array}{c}\text { Recall } \\
\text { Bias } \\
\text { (Yes/No) }\end{array}$ & $\begin{array}{l}\text { Confidence Intervals } \\
\text { (NA/Precise/Imprecise) }\end{array}$ & $\begin{array}{l}\text { Analytical Methods to Adjust for } \\
\text { Confounding }\end{array}$ \\
\hline $\begin{array}{c}\text { Alaimo } \\
\text { et al. (2001) } \\
\text { [73] }\end{array}$ & $\begin{array}{l}\text { Observational, } \\
\text { cross- } \\
\text { sectional, } \\
\text { secondary } \\
\text { data }\end{array}$ & $\begin{array}{l}\text { Oversampling of } \\
\text { subgroups } \\
\text { Representative } \\
\text { sample } \\
\text { Sampling weights }\end{array}$ & $+1-$ & Yes & NA & $\begin{array}{l}\text { Main effect linear and logistic } \\
\text { models, adjusted for } \\
\text { sociodemographics and health } \\
\text { outcomes: blood lead concentration, } \\
\text { family size, mother's age at birth, } \\
\text { presence of birth complications, low } \\
\text { birth weight, prenatal smoke } \\
\text { exposure, parental perceptions of } \\
\text { child's health status }\end{array}$ \\
\hline $\begin{array}{l}\text { Barnes et al. } \\
(2012)[83]\end{array}$ & $\begin{array}{l}\text { Observational, } \\
\text { longitudinal, } \\
\text { primary data }\end{array}$ & None & $+1-$ & Yes & NA & $\begin{array}{l}\text { Mixed-effect models, adjusted for } \\
\text { sociodemographics, medical } \\
\text { conditions, geographic region, age, } \\
\text { race, sex, years of educational } \\
\text { attainment, self-reported myocardial } \\
\text { infarction, hypertension, stroke, } \\
\text { diabetes, southern vs. other states }\end{array}$ \\
\hline
\end{tabular}


Table 3. Cont.

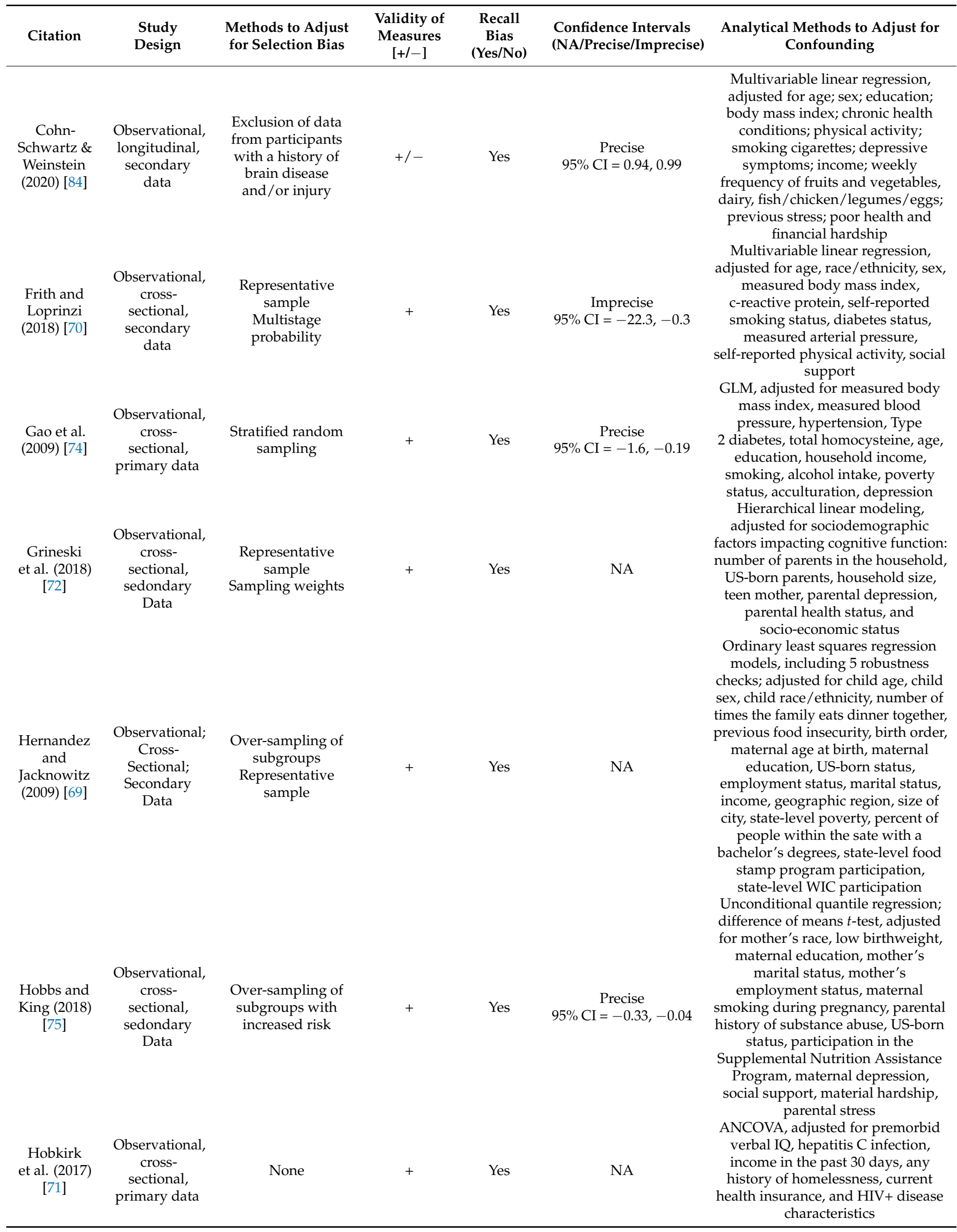


Table 3. Cont.

\begin{tabular}{|c|c|c|c|c|c|c|}
\hline Citation & $\begin{array}{l}\text { Study } \\
\text { Design }\end{array}$ & $\begin{array}{l}\text { Methods to Adjust } \\
\text { for Selection Bias }\end{array}$ & $\begin{array}{c}\text { Validity of } \\
\text { Measures } \\
{[+/-]}\end{array}$ & $\begin{array}{c}\text { Recall } \\
\text { Bias } \\
\text { (Yes/No) }\end{array}$ & $\begin{array}{l}\text { Confidence Intervals } \\
\text { (NA/Precise/Imprecise) }\end{array}$ & $\begin{array}{l}\text { Analytical Methods to Adjust for } \\
\text { Confounding }\end{array}$ \\
\hline $\begin{array}{c}\text { Koyanagi } \\
\text { et al. (2019) } \\
{[76]}\end{array}$ & $\begin{array}{l}\text { Observational, } \\
\text { cross- } \\
\text { sectional, } \\
\text { sedondary } \\
\text { Data }\end{array}$ & $\begin{array}{l}\text { Representative } \\
\text { sample } \\
\text { Stratified } \\
\text { multistage cluster } \\
\text { sampling design }\end{array}$ & $+1-$ & Yes & $\begin{array}{c}\text { Precise } \\
95 \% \mathrm{CI}=1.63,3.87\end{array}$ & $\begin{array}{l}\text { Multivariable logistic regression, } \\
\text { adjusted for sex, age, education, } \\
\text { income, race, physical activity, } \\
\text { smoking use, alcohol use, } \\
\text { depression in past } 12 \text { months, } \\
\text { measured body mass index, stroke, } \\
\text { diabetes, hypertension }\end{array}$ \\
\hline $\begin{array}{c}\text { Mayston } \\
\text { et al. (2015) } \\
{[77]}\end{array}$ & $\begin{array}{l}\text { Observational, } \\
\text { cross- } \\
\text { sectional, } \\
\text { primary data }\end{array}$ & None & $+1-$ & Yes & $\begin{array}{c}\text { Precise } \\
95 \% \mathrm{CI}=1.05,1.88\end{array}$ & $\begin{array}{c}\text { Multivariate logistic regression, } \\
\text { adjusted for sex, age, psychological } \\
\text { comorbidity; hazardous drinking } \\
\text { was adjusted for men only }\end{array}$ \\
\hline $\begin{array}{c}\text { Momtaz } \\
\text { et al. (2015) } \\
{[78]}\end{array}$ & $\begin{array}{l}\text { Observational, } \\
\text { cross- } \\
\text { sectional, } \\
\text { secondary } \\
\text { data }\end{array}$ & Random sampling & $+/-$ & Yes & $\begin{array}{c}\text { Precise } \\
95 \% \mathrm{CI}=1.13,2.92\end{array}$ & $\begin{array}{l}\text { Multiple logistic regression, adjusted } \\
\text { for age, sex, marital status, } \\
\text { educational attainment, ethnicity, } \\
\text { and place of residence }\end{array}$ \\
\hline $\begin{array}{c}\text { Onadja } \\
\text { et al. (2013) } \\
\text { [79] }\end{array}$ & $\begin{array}{l}\text { Observational, } \\
\text { cross- } \\
\text { sectional, } \\
\text { secondary } \\
\text { data }\end{array}$ & None & + & Yes & $\begin{array}{c}\text { Precise } \\
95 \% \mathrm{CI}=1.06,3.06\end{array}$ & $\begin{array}{l}\text { Linear regression, adjusted for age, } \\
\text { sex, ethnicity, socioeconomic } \\
\text { conditions in childhood, self-rated } \\
\text { health during childhood, current } \\
\text { socioeconomic status including } \\
\text { marital status, body mass index, } \\
\text { hypertension }\end{array}$ \\
\hline $\begin{array}{l}\text { Portela- } \\
\text { Parra and } \\
\text { Leung } \\
(2019)[80]\end{array}$ & $\begin{array}{l}\text { Observational, } \\
\text { cross- } \\
\text { sectional, } \\
\text { secondary } \\
\text { data }\end{array}$ & None & + & Yes & $\begin{array}{c}\text { Precise } \\
95 \% \mathrm{CI}=-0.33,-0.15 \\
95 \% \mathrm{CI}=-0.25 \\
-0.002 \\
95 \% \mathrm{CI}=-0.26,-0.01\end{array}$ & $\begin{array}{l}\text { Multivariable linear regression, } \\
\text { adjusted for age, sex, race/ethnicity, } \\
\text { highest education level, marital } \\
\text { status, income, smoking status }\end{array}$ \\
\hline $\begin{array}{l}\text { Tan et al. } \\
(2020)[85]\end{array}$ & $\begin{array}{l}\text { Observational, } \\
\text { cross- } \\
\text { sectional, } \\
\text { secondary } \\
\text { data }\end{array}$ & $\begin{array}{l}\text { Recruitment of } \\
\text { demographically } \\
\text { similar control } \\
\text { participants }\end{array}$ & + & Yes & NA & $\begin{array}{l}\text { Multivariable linear regression, } \\
\text { adjusted for income, employment } \\
\text { status, illicit and non-illicit substance } \\
\text { use, body mass index, depression, } \\
\text { post-traumatic stress disorder }\end{array}$ \\
\hline $\begin{array}{l}\text { Tong et al. } \\
\text { (2018) [81] }\end{array}$ & $\begin{array}{l}\text { Observational, } \\
\text { cross- } \\
\text { sectional, } \\
\text { secondary } \\
\text { data }\end{array}$ & Random sampling & $+1-$ & Yes & $\begin{array}{c}\text { Precise } \\
95 \% \mathrm{CI}=1.12,4.35\end{array}$ & $\begin{array}{c}\text { Logistic regression, adjusted for age, } \\
\text { race/ethnicity, employment, } \\
\text { residential history, health status, } \\
\text { depressive symptoms, smoking } \\
\text { status, alcohol use, illicit substance } \\
\text { use, social support }\end{array}$ \\
\hline $\begin{array}{l}\text { Wong et al. } \\
\text { (2016) [82] }\end{array}$ & $\begin{array}{l}\text { Observational, } \\
\text { longitudinal, } \\
\text { primary data }\end{array}$ & Random sampling & + & Yes & $\begin{array}{c}\text { Precise } \\
95 \% \mathrm{CI}=-2.19,-0.20 \\
\text { Imprecise } \\
95 \% \mathrm{CI}=-8.52,-0.85 \\
95 \% \mathrm{CI}=-8.92,-3.43\end{array}$ & $\begin{array}{c}\text { Logistic regression, adjusted for age, } \\
\text { sex, body mass index, education, } \\
\text { physical activity score, poverty, } \\
\text { acculturation score, smoking status, } \\
\text { alcohol use, overall diet quality, } \\
\text { presence of hypertension or diabetes, } \\
\text { plasma homocysteine concentration, } \\
\text { APOE status, depression, relevant } \\
\text { baseline cognitive test score, time } \\
\text { between assessments }\end{array}$ \\
\hline
\end{tabular}

$+=$ strong validity; - = weak validity; +/ - = mixed validity assessment of bias and strength of findings.

\section{Discussion}

This systematic review explored the association between food insecurity and cognitive function across the life course. It is critical to understand the association between food insecurity and cognitive function to better understand how different forms of adversity are related to cognitive function and to examine the downstream effects of this relationship on other negative health outcomes (e.g., cognitive impairment, obesity, stress). Our results are in accord with Na et al.'s [60] review among adults, while expanding the findings by spanning the human life course through including four studies among children $[69,72,73,75]$ and four adult studies not included in the aforementioned review $[71,80,84,85]$. The findings in our review also broaden the evidence of a significant association between food insecurity and cognitive function by confirming the findings from other relatively similar 
reviews delineating the impact of poor social conditions, such as food insecurity, on the well-being of children [19] and adults [20]. However, it is important to note that many of the studies indicated modest effect sizes, especially when the cognitive function was a continuous outcome.

Differences in general cognitive abilities such as attention, orientation, associative learning, and perceptual speed by food security status were assessed in almost every study of middle- or older adults identified in this review [70,71,74,76,78-83,85]. Tests that assess general cognition may indicate moderate to severe forms of cognitive dysfunction that may affect daily living and quality of life [88-90]. For example, cognitive impairment may affect relationships with others and, in some cases, may impair a person's abilities to live independently [91]. Given that general cognitive faculties are known to decline with age [92,93], the evidence of associations between food insecurity and general cognitive decline is particularly worrisome for older adults, as food insecurity may pose a compounding burden on cognitive health. For those experiencing food insecurity, issues of endogeneity may be at play in the relationships observed in the reviewed studies; however, most studies adjusted for socio-cultural variables often associated with food insecurity such as education, poverty/income, maternal age at birth, or race/ethnicity.

While cognitive function among middle-aged and older adults with food insecurity has been discussed previously [60], our review yields novel study findings indicating significant inverse associations between food insecurity and general cognitive function in studies of toddlers and young children $[69,75]$. Impaired cognitive development in children is associated with behavioral issues such as irritability, impatience, and distractibility [94]. Related evidence has established links between food insecurity and behavioral issues in children (e.g., aggression, anxiety, hyperactivity) [95-98], thus raising the question of whether the impairment of cognitive development is a mediating factor in such associations. Only one study, to our knowledge, provided credible evidence of an association between food insecurity and impaired cognitive development in children [75], thus highlighting a need for further research in order to better understand how food insecurity impacts human development in children.

Executive function, another commonly explored aspect of cognitive function, involves higher-order cognitive processes allowing individuals to plan, regulate behavior, and achieve goals [99]. Related research has shown that food insecurity is associated with aspects of children's academic performance [12,96], and executive functions such as working memory abilities are known predictors of academic performance [100]. The impairment of working memory may play a role in the association between food insecurity and academic outcomes, although no research has directly explored this possibility. However, it has been proposed that the association between food insecurity and impaired executive functioning in both children and adults may result from the effects of unhealthy stress on the prefrontal cortex [101,102], an essential brain region for carrying out executive functions [103]. Future research is needed to examine the relative effects of factors associated with cognitive functioning such as stress and food insecurity.

An unexpected finding in our review is that moderate food insecurity may have a greater impact on cognitive function compared with greater levels of food insecurity [76]. This observation is similar to results from previous research, where acute food insecurity had a greater impact on behavioral outcomes among children than chronic food insecurity $[69,72]$, which might be explained in terms of the impact of stress on those with acute versus chronic food insecurity. Individuals suffering from chronic food insecurity might develop coping strategies for dealing with longer-term food insecurity, while those with acute food insecurity experiences may be less resilient due their volatile food security status. Acute food insecurity could involve less predictable circumstances than chronic food insecurity, and it is possible for such unpredictable circumstances to induce greater stress compared with more familiar situations [104]. Future research is needed to confirm how chronic and acute food insecurity are associated with cognitive functioning. However, it is unknown how altering food insecurity alone may impact cognitive function. Perhaps 
it is only when we are able to address the multifactorial root causes of social determinants of health that impact chronic and acute stress such as food insecurity, we will be able to see improvements in cognitive function for vulnerable populations.

Given that most studies included in the review were secondary analyses of large, representative datasets, studies were able to consider several confounding variables in their analyses related to both food insecurity and cognitive function, most consistently sociodemographics, depression, health behaviors (e.g., smoking), and body mass index. However, the inclusion of specific confounders was not consistent, nor were they exhaustive of factors that are related for to food insecurity. For example, research has indicated that adverse childhood experiences are related to food insecurity $[105,106]$ and, independently, cognitive function $[107,108]$. Other research has linked structural racism independently from food insecurity $[109,110]$ and cognitive function [111]. Importantly, food insecurity may be a proxy for other individual and societal factors not assessed in the studies included in this review. Research is needed to explore the relative impact of food insecurity and other forms of adversity on cognitive function. Further, all studies were observational. While some studies were longitudinal, no studies were able to address all aspects of causal inference. It is possible that reverse causality is at play, where cognitive function impacts the ability to consistently access food; however, all 17 studies examined in the current review assessed their hypothesis in one direction: food insecurity as the independent variable and the cognitive outcome(s) as the dependent variable. Further research is needed to examine the possible causal pathway involved in the association between food insecurity and various types of cognitive function.

\section{Strengths and Limitations}

This systematic review has certain strengths and limitations that should be considered when interpreting the findings. One strength involved our assessment of bias, which included select items from the Preliminary Tool for Risk of Bias in Exposure Studies [65], Q-Coh [66], and tools for grading the strength and quality of a body of evidence [67,68]. These tools permitted the investigators to perform comprehensive assessments of biases within the included studies to determine the validity and reliability of the study findings. This study included every age group across the life course, as this approach permitted the investigators to compile all findings that were relevant to the association between food insecurity and cognitive function, and to proceed to establish a comprehensive understanding of what is already known about this relationship while subsequently articulating the knowledge gaps that still need to be addressed by research. However, because such a variety of measures of cognitive function were examined, a meta-analysis was not possible. A limitation of this study was the inclusion of studies with participants whose health and socioeconomic status confounded their measured food security and cognitive functioning (e.g., older adults with dementia, homeless individuals). However, these studies were included to better understand food insecurity experiences among people living with diverse conditions. An additional limitation is that most studies in our review using secondary data for statistical analyses, which poses study design restrictions for researchers concerning participant sampling, construct measure selection, and data collection approaches. Furthermore, the generalizability of the findings from study to study in this review is uncertain due to the multitude of different measures used to examine the same constructs.

\section{Conclusions}

The findings from this review indicate that food insecurity status correlates with poorer cognitive function across the life course. Our review corroborates the findings from a recently published review detailing an inverse relationship between food insecurity and both executive functioning and verbal memory among middle-aged and older adults. Our expansive life course review provides critical evidence detailing how children with food insecurity may suffer from delayed cognitive development, which could negatively impact academic performance while increasing the risk of poorer health outcomes later in life. 
There is a need for further research exploring the associations between food insecurity and cognitive function among certain populations (e.g., adolescents, young adults, college students). Research is also needed that objectively measures brain structure and function to more fully understand the ramifications of food insecurity experiences. Overall, the evidence from this review highlights a present and critical need for nutrition and public health interventions aimed at preventing food insecurity and cognitive decline.

Supplementary Materials: The following are available online at https:/ / www.mdpi.com/article/10 .3390 /ijtm1030015/s1. Table S1: Comprehensive list of search terms to identify literature assessing the relationship between food insecurity and cognitive function.

Author Contributions: M.F.R.: conducting the database searches, article screening, data extraction, manuscript writing, table and figure creation; N.G.: article screening, data extraction, manuscript writing, table and figure creation; B.B.B. and M.N.L.: article screening, data accuracy checks, and editorial comments; M.B.; study design and supervision, article screening, data accuracy checks, manuscript writing, and editorial comments. All authors have read and agreed to the published version of the manuscript.

Funding: This manuscript was supported by an internal grant from the College of Health Solutions at Arizona State University (grant number PG13046; MPIs Bruening and Braden).

Institutional Review Board Statement: Not applicable.

Informed Consent Statement: Not applicable.

Data Availability Statement: Not applicable.

Conflicts of Interest: The authors declare that they have no conflict of interest.

\section{References}

1. United States Department of Agriculture (USDA)-Economic Research Service. Definitions of Food Security. Available online: https:/ / www.ers.usda.gov/topics/food-nutrition-assistance/food-security-in-the-us / definitions-of-food-security.aspx (accessed on 1 September 2021).

2. Coleman Jensen, A.; Rabbitt, M.P.; Gregory, C.A.; Singh, A. Household Food Security in the United States in 2019; United States Department of Agriculture: Washington, DC, USA, 2020; p. 47.

3. Drennen, C.R.; Coleman, S.M.; de Cuba, S.E.; Frank, D.A.; Chilton, M.; Cook, J.T.; Cutts, D.B.; Heeren, T.; Casey, P.H.; Black, M.M. Food Insecurity, Health, and Development in Children Under Age Four Years. Pediatrics 2019, 144, e20190824. [CrossRef]

4. Frongillo, E. Advancing Knowledge of How and Why Food Insecurity Is Associated with Poor Well-Being in Families and Individuals across the Life Course. J. Acad. Nutr. Diet. 2019, 119, 1621-1622. [CrossRef]

5. Phojanakong, P.; Brown-Weida, E.; Grimaldi, G.; Lê-Scherban, F.; Chilton, M. Experiences of Racial and Ethnic Discrimination Are Associated with Food Insecurity and Poor Health. Int. J. Environ. Res. Public Health 2019, 16, 4369. [CrossRef] [PubMed]

6. Gundersen, C.; Ziliak, J.P. Food insecurity and health outcomes. Health Aff. 2015, 34, 1830-1839. [CrossRef] [PubMed]

7. Gundersen, C.; Kreider, B. Bounding the effects of food insecurity on children's health outcomes. J. Health Econ. 2009, 28, 971-983. [CrossRef] [PubMed]

8. Ashiabi, G. Household food insecurity and children's school engagement. J. Child. Poverty 2005, 11, 3-17. [CrossRef]

9. Whitaker, R.C.; Phillips, S.M.; Orzol, S.M. Food insecurity and the risks of depression and anxiety in mothers and behavior problems in their preschool-aged children. Pediatrics 2006, 118, e859-e868. [CrossRef]

10. McIntyre, L.; Williams, J.V.; Lavorato, D.H.; Patten, S. Depression and suicide ideation in late adolescence and early adulthood are an outcome of child hunger. J. Affect. Disord. 2013, 150, 123-129. [CrossRef]

11. Huang, J.; Matta Oshima, K.M.; Kim, Y. Does food insecurity affect parental characteristics and child behavior? Testing mediation effects. Soc. Serv. Rev. 2010, 84, 381-401. [CrossRef]

12. Jyoti, D.F.; Frongillo, E.A.; Jones, S.J. Food insecurity affects school children's academic performance, weight gain, and social skills. J. Nutr. 2005, 135, 2831-2839. [CrossRef]

13. Esfandiari, S.; Omidvar, N.; Eini-Zinab, H.; Doustmohammadian, A.; Amirhamidi, Z. Associations Among Food Insecurity, Academic Performance, and Weight Status in Primary Schoolchildren in Tehran, Iran: A Cross-sectional Study. J. Nutr. Educ. Behav. 2018, 50, 109-117. [CrossRef]

14. Winicki, J.; Jemison, K. Food insecurity and hunger in the kindergarten classroom: Its effect on learning and growth. Contemp. Econ. Policy 2003, 21, 145-157. [CrossRef]

15. Vozoris, N.T.; Tarasuk, V.S. Household food insufficiency is associated with poorer health. J. Nutr. 2003, 133, 120-126. [CrossRef]

16. Seligman, H.K.; Laraia, B.A.; Kushel, M.B. Food insecurity is associated with chronic disease among low-income NHANES participants. J. Nutr. 2009, 140, 304-310. [CrossRef] [PubMed] 
17. Ding, M.; Keiley, M.K.; Garza, K.B.; Duffy, P.A.; Zizza, C.A. Food insecurity is associated with poor sleep outcomes among US adults. J. Nutr. 2014, 145, 615-621. [CrossRef] [PubMed]

18. Martin, M.; Maddocks, E.; Chen, Y.; Gilman, S.; Colman, I. Food insecurity and mental illness: Disproportionate impacts in the context of perceived stress and social isolation. Public Health 2016, 132, 86-91. [CrossRef] [PubMed]

19. Chaudry, A.; Wimer, C. Poverty is not just an indicator: The relationship between income, poverty, and child well-being. Acad. Pediatr. 2016, 16, S23-S29. [CrossRef] [PubMed]

20. Durao, S.; Visser, M.E.; Ramokolo, V.; Oliveira, J.M.; Schmidt, B.-M.; Balakrishna, Y.; Brand, A.; Kristjansson, E.; Schoonees, A. Community-level interventions for improving access to food in low-and middle-income countries. Cochrane Database Syst. Rev. 2020, 7, CD011504. [CrossRef]

21. Champakam, S.; Srikantia, S.; Gopalan, C. Kwashiorkor and mental development. Am. J. Clin. Nutr. 1968, 21, 844-852. [CrossRef] [PubMed]

22. Hoorweg, J.C. Protein-Energy Malnutrition and Intellectual Abilities: A Study of Teen-Age Ugandan Children; Walter de Gruyter GmbH \& Co KG: Berlin, Germany, 2019; Volume 5.

23. Galler, J.R.; Ramsey, F. A follow-up study of the influence of early malnutrition on development: V. Delayed development of conservation (Piaget). J. Am. Acad. Child Adolesc. Psychiatry 1987, 26, 23-27. [CrossRef]

24. Upadhyay, S.; Agarwal, D.; Shastri, J.; Agarwal, K. Persistence of soft neurological signs in chronic undernourished children. Nutr. Res. 1995, 15, 193-199. [CrossRef]

25. Martorell, R. Undernutrition during pregnancy and early childhood and its consequences for cognitive and behavioral development. Early Child Dev. Investig. Our Child. Future 1997, 39-83.

26. Mendez, M.A.; Adair, L.S. Severity and timing of stunting in the first two years of life affect performance on cognitive tests in late childhood. J. Nutr. 1999, 129, 1555-1562. [CrossRef] [PubMed]

27. Kar, B.R.; Rao, S.L.; Chandramouli, B. Cognitive development in children with chronic protein energy malnutrition. Behav. Brain Funct. 2008, 4, 31. [CrossRef] [PubMed]

28. De Rooij, S.R.; Wouters, H.; Yonker, J.E.; Painter, R.C.; Roseboom, T.J. Prenatal undernutrition and cognitive function in late adulthood. Proc. Natl. Acad. Sci. USA 2010, 107, 16881-16886. [CrossRef]

29. Misra, U.; Kalital, J.; Kumar, S.; Poptani, H.; Agarwal, D.; Agarwal, K. Brain MRI and cognitive evoked potentials in rural chronically undernourished children. Nutr. Res. 1996, 16, 1147-1151. [CrossRef]

30. Agarwal, K.; Das, D.; Agarwal, D.; Upadhyay, S.; Mishra, S. Soft neurological signs and EEG pattern in rural malnourished children. Acta Pædiatr. 1989, 78, 873-878. [CrossRef]

31. Benítez-Bribiesca, L.; De la Rosa-Alvarez, I.; Mansilla-Olivares, A. Dendritic spine pathology in infants with severe protein-calorie malnutrition. Pediatrics 1999, 104, e21. [CrossRef]

32. Hay, P.J.; Sachdev, P. Brain dysfunction in anorexia nervosa: Cause or consequence of under-nutrition? Curr. Opin. Psychiatry 2011, 24, 251-256. [CrossRef] [PubMed]

33. Sarrar, L.; Holzhausen, M.; Warschburger, P.; Pfeiffer, E.; Lehmkuhl, U.; Schneider, N. Cognitive function in adolescent patients with anorexia nervosa and unipolar affective disorders. Eur. Eat. Disord. Rev. 2016, 24, 232-240. [CrossRef]

34. Jones, B.P.; Duncan, C.C.; Brouwers, P.; Mirsky, A.F. Cognition in eating disorders. J. Clin. Exp. Neuropsychol. 1991, 13, 711-728. [CrossRef] [PubMed]

35. Martin, C.K.; Anton, S.D.; Han, H.; York-Crowe, E.; Redman, L.M.; Ravussin, E.; Williamson, D.A. Examination of cognitive function during six months of calorie restriction: Results of a randomized controlled trial. Rejuvenation Res. 2007, 10, 179-190. [CrossRef] [PubMed]

36. Solianik, R.; Sujeta, A.; Čekanauskaitè, A. Effects of 2-day calorie restriction on cardiovascular autonomic response, mood, and cognitive and motor functions in obese young adult women. Exp. Brain Res. 2018, 236, 2299-2308. [CrossRef]

37. Green, M.W.; Elliman, N.A.; Rogers, P.J. The effects of food deprivation and incentive motivation on blood glucose levels and cognitive function. Psychopharmacology 1997, 134, 88-94. [CrossRef]

38. Lieberman, H.R.; Caruso, C.M.; Niro, P.J.; Adam, G.E.; Kellogg, M.D.; Nindl, B.C.; Kramer, F.M. A double-blind, placebocontrolled test of $2 \mathrm{~d}$ of calorie deprivation: Effects on cognition, activity, sleep, and interstitial glucose concentrations. Am. J. Clin. Nutr. 2008, 88, 667-676. [CrossRef] [PubMed]

39. Giles, G.E.; Mahoney, C.R.; Caruso, C.; Bukhari, A.S.; Smith, T.J.; Pasiakos, S.M.; McClung, J.P.; Lieberman, H.R. Two days of calorie deprivation impairs high level cognitive processes, mood, and self-reported exertion during aerobic exercise: $\mathrm{A}$ randomized double-blind, placebo-controlled study. Brain Cogn. 2019, 132, 33-40. [CrossRef]

40. Witte, A.; Fobker, M.; Gellner, R.; Knecht, S.; Flöel, A. Caloric restriction improves memory in elderly humans. Proc. Natl. Acad. Sci. USA 2009, 106, 1255-1260. [CrossRef] [PubMed]

41. Benau, E.M.; Orloff, N.C.; Janke, E.A.; Serpell, L.; Timko, C.A. A systematic review of the effects of experimental fasting on cognition. Appetite 2014, 77, 52-61. [CrossRef]

42. Wells, J.C.; Sawaya, A.L.; Wibaek, R.; Mwangome, M.; Poullas, M.S.; Yajnik, C.S.; Demaio, A. The double burden of malnutrition: Aetiological pathways and consequences for health. Lancet 2020, 395, 75-88. [CrossRef]

43. Subramanian, S.; Kawachi, I.; Smith, G.D. Income inequality and the double burden of under-and overnutrition in India. J. Epidemiol. Community Health 2007, 61, 802-809. [CrossRef] 
44. Dabelea, D.; Harrod, C.S. Role of developmental overnutrition in pediatric obesity and type 2 diabetes. Nutr. Rev. 2013, 71, S62-S67. [CrossRef]

45. Samartín, S.; Chandra, R.K. Obesity, overnutrition and the immune system. Nutr. Res. 2001, 21, 243-262. [CrossRef]

46. Gupta, D.; Krueger, C.B.; Lastra, G. Over-nutrition, obesity and insulin resistance in the development of $\beta$-cell dysfunction. Curr. Diabetes Rev. 2012, 8, 76-83. [CrossRef] [PubMed]

47. Mozaffarian, D.; Fleischhacker, S.; Andrés, J.R. Prioritizing nutrition security in the US. JAMA 2021, 325, 1605-1606. [CrossRef] [PubMed]

48. Nguyen, B.T.; Shuval, K.; Bertmann, F.; Yaroch, A.L. The Supplemental Nutrition Assistance Program, food insecurity, dietary quality, and obesity among US adults. Am. J. Public Health 2015, 105, 1453-1459. [CrossRef] [PubMed]

49. Hanson, K.L.; Connor, L.M. Food insecurity and dietary quality in US adults and children: A systematic review. Am. J. Clin. Nutr. 2014, 100, 684-692. [CrossRef]

50. Robaina, K.A.; Martin, K.S. Food insecurity, poor diet quality, and obesity among food pantry participants in Hartford, CT. J. Nutr. Educ. Behav. 2013, 45, 159-164. [CrossRef]

51. Moradi, S.; Mirzababaei, A.; Dadfarma, A.; Rezaei, S.; Mohammadi, H.; Jannat, B.; Mirzaei, K. Food insecurity and adult weight abnormality risk: A systematic review and meta-analysis. Eur. J. Nutr. 2019, 58, 45-61. [CrossRef]

52. Min, J.; Zhao, Y.; Slivka, L.; Wang, Y. Double burden of diseases worldwide: Coexistence of undernutrition and overnutritionrelated non-communicable chronic diseases. Obes. Rev. 2018, 19, 49-61. [CrossRef]

53. Lourida, I.; Soni, M.; Thompson-Coon, J.; Purandare, N.; Lang, I.A.; Ukoumunne, O.C.; Llewellyn, D.J. Mediterranean diet, cognitive function, and dementia: A systematic review. Epidemiology 2013, 4, 479-489. [CrossRef]

54. Hossain, S.; Beydoun, M.A.; Weiss, J.; Kuczmarski, M.F.; Evans, M.K.; Zonderman, A.B. Longitudinal associations between dietary quality and Alzheimer's Disease genetic risk on cognitive performance among African American adults. Br. J. Nutr. 2020, 124, 1264-1276. [CrossRef]

55. Sarker, G.; Peleg-Raibstein, D. Maternal overnutrition induces long-term cognitive deficits across several generations. Nutrients 2019, 11, 7. [CrossRef] [PubMed]

56. Fielding, R.A.; Gunstad, J.; Gustafson, D.R.; Heymsfield, S.B.; Launer, L.J.; Kral, J.G.; Penninger, J.; Phillips, D.I.; Scarmeas, N. The paradox of overnutrition in aging and cognition. Ann. N. Y. Acad. Sci. 2013, 1287, 31. [CrossRef] [PubMed]

57. Dinour, L.M.; Bergen, D.; Yeh, M.-C. The food insecurity-obesity paradox: A review of the literature and the role food stamps may play. J. Am. Diet. Assoc. 2007, 107, 1952-1961. [CrossRef]

58. Hernandez, D.C.; Reesor, L.; Murillo, R. Gender disparities in the food insecurity-overweight and food insecurity-obesity paradox among low-income older adults. J. Acad. Nutr. Diet. 2017, 117, 1087-1096. [CrossRef] [PubMed]

59. World Health Organization. A Life Course Approach to Health. Available online: https://www.who.int/ageing/publications/ lifecourse/alc_lifecourse_training_en.pdf (accessed on 1 September 2021).

60. Na, M.; Dou, N.; Ji, N.; Xie, D.; Huang, J.; Tucker, K.L.; Gao, X. Food insecurity and cognitive function in middle to older adulthood: A systematic review. Adv. Nutr. 2020, 11, 667-676. [CrossRef]

61. Fávaro-Moreira, N.C.; Krausch-Hofmann, S.; Matthys, C.; Vereecken, C.; Vanhauwaert, E.; Declercq, A.; Bekkering, G.E.; Duyck, J. Risk factors for malnutrition in older adults: A systematic review of the literature based on longitudinal data. Adv. Nutr. 2016, 7, 507-522. [CrossRef]

62. Smith, K.E.; Mason, T.B.; Johnson, J.S.; Lavender, J.M.; Wonderlich, S.A. A systematic review of reviews of neurocognitive functioning in eating disorders: The state-of-the-literature and future directions. Int. J. Eat. Disord. 2018, 51, 798-821. [CrossRef]

63. Siervo, M.; Arnold, R.; Wells, J.; Tagliabue, A.; Colantuoni, A.; Albanese, E.; Brayne, C.; Stephan, B. Intentional weight loss in overweight and obese individuals and cognitive function: A systematic review and meta-analysis. Obes. Rev. 2011, 12, 968-983. [CrossRef]

64. Weerahandi, S. Generalized confidence intervals. In Exact Statistical Methods for Data Analysis; Springer: Berlin/Heidelberg, Germany, 1995; pp. 143-168.

65. Viswanathan, M.; Patnode, C.D.; Berkman, N.D.; Bass, E.B.; Chang, S.; Hartling, L.; Murad, M.H.; Treadwell, J.R.; Kane, R.L. Assessing the Risk of Bias in Systematic Reviews of Health Care Interventions. Methods Guide for Effectiveness and Comparative Effectiveness Reviews [Internet]. 2017. Available online: https://www.ncbi.nlm.nih.gov/sites/books/NBK519366/ (accessed on 13 August 2021).

66. Jarde, A.; Losilla, J.-M.; Vives, J.; Rodrigo, M.F. Q-Coh: A tool to screen the methodological quality of cohort studies in systematic reviews and meta-analyses. Int. J. Clin. Health Psychol. 2013, 13, 138-146. [CrossRef]

67. Owens, D.K.; Lohr, K.N.; Atkins, D.; Treadwell, J.R.; Reston, J.T.; Bass, E.B.; Chang, S.; Helfand, M. Grading the strength of a body of evidence when comparing medical interventions. In Methods Guide for Effectiveness and Comparative Effectiveness Reviews [Internet]; Agency for Healthcare Research and Quality (US): Rockville, MD, USA, 2009.

68. Lohr, K.N.; Carey, T.S. Assessing "best evidence": Issues in grading the quality of studies for systematic reviews. Jt. Comm. J. Qual. Improv. 1999, 25, 470-479. [CrossRef]

69. Hernandez, D.C.; Jacknowitz, A. Transient, but not persistent, adult food insecurity influences toddler development. J. Nutr. 2009, 139, 1517-1524. [CrossRef] [PubMed]

70. Frith, E.; Loprinzi, P.D. Food insecurity and cognitive function in older adults: Brief report. Clin. Nutr. 2018, 37, 1765-1768. [CrossRef] 
71. Hobkirk, A.L.; Towe, S.L.; Patel, P.; Meade, C.S. Food insecurity is associated with cognitive deficits among hiv-positive, but not hiv-negative, individuals in a united states sample. AIDS Behav. 2017, 21, 783-791. [CrossRef] [PubMed]

72. Grineski, S.E.; Morales, D.X.; Collins, T.W.; Rubio, R. Transitional dynamics of household food insecurity impact children's developmental outcomes. J. Dev. Behav. Pediatr. JDBP 2018, 39, 715. [CrossRef]

73. Alaimo, K.; Olson, C.M.; Frongillo, E.A. Food insufficiency and American school-aged children's cognitive, academic, and psychosocial development. Pediatrics 2001, 108, 44-53. [PubMed]

74. Gao, X.; Scott, T.; Falcon, L.M.; Wilde, P.E.; Tucker, K.L. Food insecurity and cognitive function in Puerto Rican adults. Am. J. Clin. Nutr. 2009, 89, 1197-1203. [CrossRef]

75. Hobbs, S.; King, C. The unequal impact of food insecurity on cognitive and behavioral outcomes among 5-year-old urban children. J. Nutr. Educ. Behav. 2018, 50, 687-694. [CrossRef]

76. Koyanagi, A.; Veronese, N.; Stubbs, B.; Vancampfort, D.; Stickley, A.; Oh, H.; Shin, J.I.; Jackson, S.; Smith, L.; Lara, E. Food insecurity is associated with mild cognitive impairment among middle-aged and older adults in South Africa: Findings from a nationally representative survey. Nutrients 2019, 11, 749. [CrossRef]

77. Mayston, R.; Patel, V.; Abas, M.; Korgaonkar, P.; Paranjape, R.; Rodrigues, S.; Prince, M. Determinants of common mental disorder, alcohol use disorder and cognitive morbidity among people coming for HIV testing in Goa, India. Trop. Med. Int. Health 2015, 20, 397-406. [CrossRef]

78. Momtaz, Y.A.; Haron, S.A.; Hamid, T.A.; Ibrahim, R.; Masud, J. Does food insufficiency in childhood contribute to dementia in later life? Clin. Interv. Aging 2015, 10, 49. [CrossRef]

79. Onadja, Y.; Atchessi, N.; Soura, B.A.; Rossier, C.; Zunzunegui, M.-V. Gender differences in cognitive impairment and mobility disability in old age: A cross-sectional study in Ouagadougou, Burkina Faso. Arch. Gerontol. Geriatr. 2013, 57, 311-318. [CrossRef]

80. Portela-Parra, E.T.; Leung, C.W. Food Insecurity Is Associated with Lower Cognitive Functioning in a National Sample of Older Adults. J. Nutr. 2019, 149, 1812-1817. [CrossRef]

81. Tong, M.; Tieu, L.; Lee, C.; Ponath, C.; Guzman, D.; Kushel, M. Factors associated with food insecurity among older homeless adults: Results from the HOPE HOME study. J. Public Health 2019, 41, 240-249. [CrossRef]

82. Wong, J.C.; Scott, T.; Wilde, P.; Li, Y.-G.; Tucker, K.L.; Gao, X. Food insecurity is associated with subsequent cognitive decline in the Boston Puerto Rican Health Study. J. Nutr. 2016, 146, 1740-1745. [CrossRef]

83. Barnes, L.L.; Wilson, R.S.; Everson-Rose, S.A.; Hayward, M.D.; Evans, D.A.; De Leon, C.F.M. Effects of early-life adversity on cognitive decline in older African Americans and whites. Neurology 2012, 79, 2321-2327. [CrossRef] [PubMed]

84. Cohn-Schwartz, E.; Weinstein, G. Early-life food deprivation and cognitive performance among older Europeans. Maturitas 2020, 141, 26-32. [CrossRef]

85. Tan, J.Y.; Sheira, L.A.; Frongillo, E.A.; Adimora, A.A.; Tien, P.C.; Konkle-Parker, D.; Golub, E.T.; Merenstein, D.; Levin, S.; Cohen, M. Food insecurity and neurocognitive function among women living with or at risk for HIV in the United States. Am. J. Clin. Nutr. 2020, 112, 1280-1286. [CrossRef] [PubMed]

86. United States Department of Agriculture (USDA)—Economic Research Service. U.S. Household Food Security Survey Module: Three-Stage Design, with Screeners. Available online: https://www.ers.usda.gov/media/8271/hh2012.pdf (accessed on 1 September 2021).

87. Wechsler, D. Wechsler Adult Intelligence Scale, 3rd ed.; Yonago Acta Medica: Yonago, Japan, 1997.

88. Creavin, S.T.; Wisniewski, S.; Noel-Storr, A.H.; Trevelyan, C.M.; Hampton, T.; Rayment, D.; Thom, V.M.; Nash, K.J.; Elhamoui, H.; Milligan, R. Mini-Mental State Examination (MMSE) for the detection of dementia in clinically unevaluated people aged 65 and over in community and primary care populations. In Cochrane Database of Systematic Reviews; John Wiley \& Sons, Ltd.: Chichester, UK, 2016.

89. Jaeger, J. Digit symbol substitution test: The case for sensitivity over specificity in neuropsychological testing. J. Clin. Psychopharmacol. 2018, 38, 513. [CrossRef]

90. Tombaugh, T.N.; McIntyre, N.J. The mini-mental state examination: A comprehensive review. J. Am. Geriatr. Soc. 1992, 40, 922-935. [CrossRef] [PubMed]

91. Mitchell, A.J.; Kemp, S.; Benito-León, J.; Reuber, M. The influence of cognitive impairment on health-related quality of life in neurological disease. Acta Neuropsychiatr. 2010, 22, 2-13. [CrossRef]

92. Bishop, N.A.; Lu, T.; Yankner, B.A. Neural mechanisms of ageing and cognitive decline. Nature 2010, 464, 529-535. [CrossRef]

93. Deary, I.J.; Corley, J.; Gow, A.J.; Harris, S.E.; Houlihan, L.M.; Marioni, R.E.; Penke, L.; Rafnsson, S.B.; Starr, J.M. Age-associated cognitive decline. Br. Med. Bull. 2009, 92, 135-152. [CrossRef]

94. Cheng, E.R.; Palta, M.; Kotelchuck, M.; Poehlmann, J.; Witt, W.P. Cognitive delay and behavior problems prior to school age. Pediatrics 2014, 134, e749-e757. [CrossRef]

95. Perez-Escamilla, F.; de Toledo Vianna, R.P. Food Insecurity and the Behavioral and Intellectual Development of Children: A Review of the Evidence. J. Appl. Res. Child. 2012, 3, 9.

96. Shankar, P.; Chung, R.; Frank, D.A. Association of food insecurity with children's behavioral, emotional, and academic outcomes: A systematic review. J. Dev. Behav. Pediatr. 2017, 38, 135-150. [CrossRef] [PubMed]

97. Slopen, N.; Fitzmaurice, G.; Williams, D.R.; Gilman, S.E. Poverty, food insecurity, and the behavior for childhood internalizing and externalizing disorders. J. Am. Acad. Child Adolesc. Psychiatry 2010, 49, 444-452. 
98. Kleinman, R.E.; Murphy, J.M.; Little, M.; Pagano, M.; Wehler, C.A.; Regal, K.; Jellinek, M.S. Hunger in children in the United States: Potential behavioral and emotional correlates. Pediatrics 1998, 101, e3. [CrossRef]

99. Banich, M.T.; Compton, R.J. Cognitive Neuroscience; Cambridge University Press: Cambridge, UK, 2018.

100. Alloway, T.P.; Alloway, R.G. Investigating the predictive roles of working memory and IQ in academic attainment. J. Exp. Child Psychol. 2010, 106, 20-29. [CrossRef] [PubMed]

101. Arnsten, A.F. Stress signalling pathways that impair prefrontal cortex structure and function. Nat. Rev. Neurosci. 2009, 10, 410-422. [CrossRef]

102. Shansky, R.M.; Lipps, J. Stress-induced cognitive dysfunction: Hormone-neurotransmitter interactions in the prefrontal cortex. Front. Hum. Neurosci. 2013, 7, 123. [CrossRef] [PubMed]

103. Miller, E.K.; Cohen, J.D. An integrative theory of prefrontal cortex function. Annu. Rev. Neurosci. 2001, 24, 167-202. [CrossRef] [PubMed]

104. Abbott, B.B.; Schoen, L.S.; Badia, P. Predictable and unpredictable shock: Behavioral measures of aversion and physiological measures of stress. Psychol. Bull. 1984, 96, 45. [CrossRef]

105. Jackson, D.B.; Chilton, M.; Johnson, K.R.; Vaughn, M.G. Adverse childhood experiences and household food insecurity: Findings from the 2016 National Survey of Children's Health. Am. J. Prev. Med. 2019, 57, 667-674. [CrossRef] [PubMed]

106. Sun, J.; Knowles, M.; Patel, F.; Frank, D.A.; Heeren, T.C.; Chilton, M. Childhood adversity and adult reports of food insecurity among households with children. Am. J. Prev. Med. 2016, 50, 561-572. [CrossRef] [PubMed]

107. Anderson-Hanley, C.; Barcelos, N.M.; Zimmerman, E.A.; Gillen, R.W.; Dunnam, M.; Cohen, B.D.; Yerokhin, V.; Miller, K.E.; Hayes, D.J.; Arciero, P.J. The Aerobic and Cognitive Exercise Study (ACES) for community-dwelling older adults with or at-risk for mild cognitive impairment (MCI): Neuropsychological, neurobiological and neuroimaging outcomes of a randomized clinical trial. Front. Aging Neurosci. 2018, 10, 76. [CrossRef]

108. Hawkins, M.A.; Layman, H.M.; Ganson, K.T.; Tabler, J.; Ciciolla, L.; Tsotsoros, C.E.; Nagata, J.M. Adverse childhood events and cognitive function among young adults: Prospective results from the national longitudinal study of adolescent to adult health. Child Abus. Negl. 2021, 115, 105008. [CrossRef]

109. Bowen, S.; Elliott, S.; Hardison-Moody, A. The structural roots of food insecurity: How racism is a fundamental cause of food insecurity. Sociol. Compass 2021, 15, e12846. [CrossRef]

110. Odoms-Young, A.M. Examining the impact of structural racism on food insecurity: Implications for addressing racial/ethnic disparities. Fam. Community Health 2018, 41, S3. [CrossRef]

111. Coogan, P.; Schon, K.; Li, S.; Cozier, Y.; Bethea, T.; Rosenberg, L. Experiences of racism and subjective cognitive function in African American women. Alzheimer's Dement. Diagn. Assess. Dis. Monit. 2020, 12, e12067. [CrossRef] [PubMed] 